Check for updates

Cite this: Nanoscale, 2021, 13, 6117

\title{
Two-dimensional Weyl points and nodal lines in pentagonal materials and their optical response $\uparrow$
}

\author{
Sergio Bravo, ${ }^{a}$ M. Pacheco, ${ }^{a}$ V. Nuñez, ${ }^{a}$ J. D. Correa (D) ${ }^{b}$ and Leonor Chico (D *c
}

Two-dimensional pentagonal structures based on the Cairo tiling are the basis of a family of layered materials with appealing physical properties. In this work we present a theoretical study of the symmetrybased electronic and optical properties of these pentagonal materials. We provide a complete classification of the space groups that support pentagonal structures for binary and ternary systems. By means of first-principles calculations, the electronic band structures and the local spin textures in momentum space are analyzed for four examples of these materials, namely, PdSeTe, PdSeS, InP 5 and $\mathrm{GeBi}_{2}$, all of which are dynamically stable. Our results show that pentagonal structures can be realized in chiral and achiral lattices with Weyl nodes pinned at high-symmetry points and nodal lines along the Brillouin zone boundary; these degeneracies are protected by the combined action of crystalline and time-reversal symmetries. Additionally, we computed the linear and nonlinear optical features of the proposed pentagonal materials and discuss some particular features such as the shift current, which shows an enhancement due to the presence of nodal lines and points, and their possible applications.

Received 5th January 2021, Accepted 16th February 2021 DOI: $10.1039 / \mathrm{d} 1 \mathrm{nr} 00064 \mathrm{k}$ rsc.li/nanoscale amount of prospective 2D materials with energetically favorable phases. In some of them, interlayer van der Waals interactions imply that they might be obtained by exfoliation. ${ }^{9}$

Among the most interesting candidates are the so-called pentagonal materials, for which the first experimental example has been synthesized recently, in the form of $\mathrm{PdSe}_{2} \cdot{ }^{12}$ The origin of pentagonal materials can be traced to the theoretical prediction of penta-graphene, ${ }^{13,14}$ and their hallmark is the geometry of their planar projection, the so-called Cairo tiling. After these seminal works, many pentagonal systems have been proposed by means of first-principles calculations, ${ }^{15-24}$ with remarkable characteristics such as excellent thermoelectrical properties, ${ }^{25}$ potential application for anode materials ${ }^{26}$ and auxetic behavior, ${ }^{27}$ among others. Also, recent experimental studies have shown that the pentagonal material $\mathrm{PdSe}_{2}$ presents good performance as an ohmic contact. ${ }^{28,29}$

Such findings have compelled us to classify systematically these pentagonal materials and their symmetry-based properties. Previous work along this line was done by H. Zhuang et al. in a series of articles. ${ }^{30-32}$ They studied all possible pentagonal tessellations of the plane (with fifteen different types of pentagons ${ }^{33}$ ) and their possible realizations in monolayer materials by first-principles calculations. They showed that the pentagonal lattice structure is feasible with only two configurations composed of two kinds of pentagons, namely, type-2 and type-4 pentagons. ${ }^{31}$ An example of the planar projection of these two structures is presented in Fig. 1. Despite of this result, no further studies have been done in order to relate 
(a)

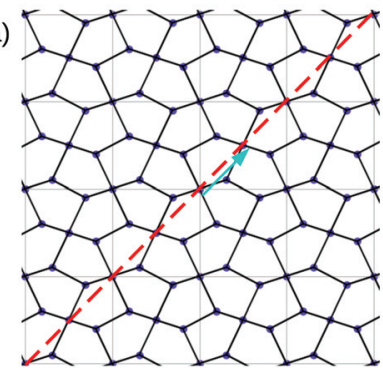

(b)

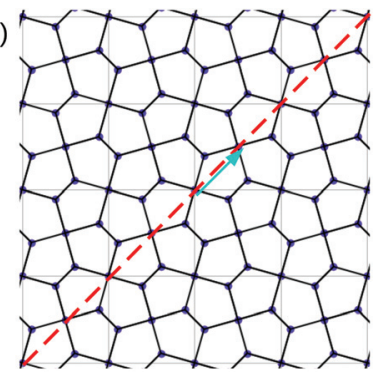

Fig. 1 Wallpaper tessellations with (a) type-2 and (b) type-4 pentagons. One of the glide planes is highlighted for each case; notice that it divides the unit cell (marked with light grey lines) along one diagonal. There is another glide plane that runs along the other diagonal. The nonsymmorphic operations consist on a mirror reflection with respect to the glide plane followed by a fractional translation depicted by the cyan vector, also along the direction determined by the intersection of the glide plane and the $2 \mathrm{D}$ projection of the pentagonal lattice.

these two possible structures with spatial groups, which would be greatly advantageous in the search for particular electronic properties linked to symmetry protection.

Thus, in this work, we classify all (layer) space groups (SG) that support unstrained pentagonal structures with up to ternary composition. We also provide a partial classification for few-layer structures with specific stackings. Further, we analyze the electronic properties constrained by crystalline symmetries. This allows us to identify nodal points and nodal lines in noncentrosymmetric SG hosting pentagonal lattices. In such cases, if a significant spin-orbit coupling (SOC) is present, it can lead to topologically nontrivial Weyl nodes pinned at high-symmetry points, as well as to nodal lines at the Brillouin zone (BZ) boundary. We present first-principles calculations for several examples of pentagonal materials supporting the group-theoretical predictions, verifying their dynamical stability for the three newly reported cases. Specifically, we address the electronic properties of PdSeTe, PdSeS, $\mathrm{InP}_{5}$ and $\mathrm{GeBi}_{2}$, which belong to three different SG. Also, with the aim to complement our findings, we provide calculations of the linear optical conductivity, natural optical activity and for the nonlinear shift current for all such examples.

This article is organized as follows. In section 2 we provide a brief description of the conditions under which the firstprinciples calculations were performed. Also the specific formulas for the optical responses are sketched. In section 3 our main results are presented. Firstly, the procedure to find all SG allowing for pentagonal structures is described. A more detailed discussion for particularly appealing SG with noncentrosymmetric character follows. Subsequently, the electronic properties of several pentagonal materials belonging to such SG are presented, providing their band structures and spin textures. To close this section, optical calculations based on the first-principles results are presented. Specifically, the linear optical conductivity, the natural rotatory power and the nonlinear shift current are reviewed, with discussing their features and possible relations to the electronic structures. Finally, in section 4 we state our conclusions and provide an outlook pin-

pointing some issues that we consider worth to be further developed in future works. Part of our results are left as ESI. $\dagger$

\section{Computational details}

The electronic properties shown in the main article of this work were computed by first-principles calculations within the GGA approximation using the PBE functional as implemented in the QUANTUM ESPRESSO package, ${ }^{34}$ with the addition of the $2 \mathrm{D}$ cutoff for layered structures. A cutoff in energy of $90 \mathrm{Ry}$ and a $17 \times 17 \times 1$ Monkhorst-Pack grid were selected, ensuring the convergence of the total energy. For relaxation purposes the force tolerance was set to $10^{-5}\left[\mathrm{eV} \AA^{-1}\right]$. We have verified our results with the GPAW first-principles package ${ }^{35}$ in order to check other hybrid functionals, obtaining a very good agreement. We have additionally confirmed the dynamical stability of the new pentagonal materials proposed here by means of the phonon dispersion relations computed with GPAW. These complementary results are shown in the ESI. $\dagger$

In the case of the optical calculations, Wannier interpolated bands were constructed, using the WANNIER90 code. ${ }^{36}$ In particular, for PdSeTe the outermost valence orbitals from each atom were included; i.e., $\mathrm{d}$ orbitals for $\mathrm{Pd}$ and $\mathrm{p}$ orbitals for Se and Te; the same type of orbitals were employed for PdSeS. For $\mathrm{InP}_{5}$, $\mathrm{p}$ valence orbitals were used for $\mathrm{P}$ and $\mathrm{sp}^{3}$ hybrid orbitals for In. In the case of $\mathrm{GeBi}_{2}$, p orbitals were used for Ge and hybrid $\mathrm{sp}^{3}$ orbitals for Bi. After the Wannier model is obtained, calculations with very dense momentum space grids can be performed. We used grids ranging from $301 \times 301 \times 1$ to $901 \times$ $901 \times 1$ in order to check convergence. Additional post processing of the band structure calculations were carried with the WannierTools package. ${ }^{37}$ Optical calculations were performed also with WANNIER90 (postw90). In particular, the optical conductivity is calculated with the Kubo formula in terms of the Berry connection, given as a function of the photon energy $\hbar \omega$ by $^{36}$

$$
\sigma_{\alpha \beta}(\hbar \omega)=\frac{i e^{2}}{\hbar N_{k} V} \sum_{k, n, m} f_{m n} E_{m n} \frac{A_{n m}^{\alpha} A_{n m}^{\beta}}{E_{m n}-(\hbar \omega+i \delta)},
$$

where $A_{n m}^{\alpha}=\left\langle u_{n}\left|i \nabla_{k \alpha}\right| u_{m}\right\rangle$ is the Berry connection and $n$ and $m$ are band indices. Also, $V$ is the unit cell volume, $N_{k}$ is the number of $k$-points in the $\mathrm{BZ}, E_{m n}=E_{m}-E_{n}\left(E_{n}=E_{n k}\right.$ is the band dispersion), $f_{m n}=f\left(E_{m k}\right)-f\left(E_{n k}\right)$, where $f\left(E_{m k}\right)$ is the Fermi-Dirac function, and $\delta$ is a parameter with units of energy. Note that the unit cell volume $V$ is that employed in the WANNIER90 code, i.e., it is a three-dimensional supercell with a large layer separation of $20 \AA$. We have verified that this value does not modify the results in a substantial way. Indeed, a variation of more than $10 \%$ on the layer separation (to $23 \AA$ ) does not alter the position of the peaks nor the general shape of the spectrum, being just a slight variation on the absolute intensity of the peaks.

The rotatory power was calculated using the expression: ${ }^{38}$

$$
\rho(\omega)=\frac{\omega^{2}}{2 c^{2}} \operatorname{Re}\left[\gamma_{x y z}(\omega)\right]
$$


where the tensor $\gamma_{\mathrm{abc}}$ is defined in terms of the band properties as $\left(\partial_{\mathrm{c}}=\partial / \partial_{k_{\mathrm{c}}}\right)^{38}$

$$
\begin{aligned}
\operatorname{Re}\left[\gamma_{\mathrm{abc}}(\omega)\right] & =\frac{e^{2}}{\varepsilon_{0} \hbar^{2}} \int[\mathrm{dk}] \sum_{n, l}^{\mathrm{occ}, \mathrm{emp}}\left[\frac{\operatorname{Re}\left(A_{\ln }^{\mathrm{b}} B_{n l}^{\mathrm{ac}}-A_{\ln }^{\mathrm{a}} B_{n l}^{\mathrm{bc}}\right)}{\omega_{\ln }{ }^{2}-\omega^{2}}\right] \\
& -\frac{3 \omega_{\ln }^{2}-\omega^{2}}{\left(\omega_{\ln }^{2}-\omega^{2}\right)^{2}} \partial_{\mathrm{c}}\left(E_{l}+E_{n}\right) \operatorname{Im}\left(A_{n l}^{\mathrm{a}} A_{n l}^{\mathrm{b}}\right) .
\end{aligned}
$$

Here $[\mathrm{dk}]=d^{\mathrm{d}} k /(2 \pi)^{\mathrm{d}}$ (with $d$ as the dimension), $\omega_{l n}=$ $\left(E_{l}-E_{n}\right) / \hbar$ and $B_{n l}^{\mathrm{ac}}$ (also valid for $B_{n l}^{\mathrm{bc}}$ ) is given by

$$
B_{n l}^{\mathrm{ac}}=B_{n l}^{\mathrm{ac} \text { ORB }}+B_{n l}^{\mathrm{ac} \text { SPIN }},
$$

where $B_{n l}^{\mathrm{acORB}}$ is the orbital contribution and $B_{n l}^{\text {acSPIN }}$ is the spin part which are respectively expressed as

$$
B_{l n}^{\mathrm{ac}(\mathrm{ORB})}=\left\langle u_{n}\left|\partial_{\mathrm{c}} H\right| \partial_{\mathrm{c}} u_{l}\right\rangle-\left\langle\partial_{\mathrm{c}} u_{n}\left|\partial_{\mathrm{c}} H\right| u_{l}\right\rangle
$$

and

$$
B_{\mathrm{ln}}^{\mathrm{ac}(\mathrm{SPIN})}=-\frac{i \hbar^{2}}{m_{\mathrm{e}}} \varepsilon_{\mathrm{abc}}\left\langle u_{n}\left|\sigma_{\mathrm{b}}\right| u_{l}\right\rangle .
$$

Finally, we compute the interband contribution to the nonlinear shift current. It relates the appearance of a current density $J_{\mathrm{a}}$ in the system due to an electric field $E$ to second order, such that $J_{\mathrm{a}}=2 \sigma_{\mathrm{abc}} \operatorname{Re}\left[E_{\mathrm{b}}^{*} E_{\mathrm{c}}\right]$, where $\sigma_{\mathrm{abc}}$ is the third rank shift current tensor. ${ }^{39}$ This is obtained using the following formula:

$$
\begin{aligned}
& \sigma_{\mathrm{abc}}(0 ; \omega,-\omega)=-\frac{i \pi e^{3}}{2 \hbar^{2}} \\
& \int[\mathrm{dk}] \sum_{n, m} f_{n m}\left(r_{m n}^{\mathrm{b}} r_{n m ; \mathrm{a}}^{\mathrm{c}}+r_{m n}^{\mathrm{c}} r_{n m ; \mathrm{a}}^{\mathrm{b}}\right) \delta\left(\omega_{m n}-\omega\right),
\end{aligned}
$$

where $r_{n m ; \mathrm{a}}^{\mathrm{b}}=\partial_{k_{\mathrm{a}}} r_{n m}^{\mathrm{b}}-i\left(A_{n n}^{\mathrm{a}}-A_{m m}^{\mathrm{a}}\right) r_{n m}^{\mathrm{b}}$ and $r_{n m}^{\mathrm{a}}=\left(1-\delta_{n m}\right) A_{n m}^{\mathrm{a}}$. The rest of the quantities are defined as in the previous formulas.

\section{Results and discussion}

\subsection{Space groups supporting pentagonal structures}

Pentagonal materials have the Cairo tiling as their two dimensional projection. The essential feature of this pattern is that it possesses two glide planes, as it can be visually appreciated in Fig. 1. This greatly constrains the spatial groups of a pentagonal structure. Therefore, we start identifying the largest tetragonal (layer) SG with this characteristic. In Table 1 we find that SG \#127, which possesses 16 symmetry operations, is the greatest layer group with this feature. ${ }^{40}$ Within this group, we can search for all the subgroups that preserve this two-glide-plane condition. Since we are only using the lattice symmetry, we are implicitly assuming that all atomic positions are equal. However, this condition can be relaxed by considering that the spatial symmetry classifies the atomic sites in different Wyckoff positions. ${ }^{41}$

Thus, in order to preserve a SG we must only care about having equal elements within each WP. As it is well-known, the basic pentagonal structure comprises two WPs, namely, a
Table 1 Space groups that can support pentagonal structures with one and two elements. The Wyckoff positions used to conform the structure are given in the third column

\begin{tabular}{lll}
\hline Group label (\#) & Point group & Wyckoff positions \\
\hline$P 4 / m b m(\# 127)$ & $4 / m m m$ & $(4 e, 2 a)$ \\
$P \overline{4} b 2(\# 117)$ & $4 / m m m$ & $(4 e, 2 a)$ \\
$P \overline{4} 21 m(\# 113)$ & $\overline{4} 2 m$ & $(4 d, 2 a)$ \\
$P 4 b m(\# 100)$ & $4 m m$ & $(4 c, 2 a)$ \\
$P 42_{1} 2(\# 90)$ & 422 & $(4 d, 2 b)$ \\
$P b a m(\# 55)$ & $m m m$ & $(4 e, 2 a)$ \\
$P b a 2(\# 32))$ & $m m 2$ & $(4 c, 2 a)$ \\
$P 2_{1} 212(\# 18) P 2_{1} 2_{1} 2(\# 18)$ & 222 & $(4 c, 2 a)$ \\
$P 2_{1} / c(\# 14)$ & $2 / m$ & $(4 e, 2 a)$ \\
$C m(\# 8)$ & $m$ & $(4 b, 2 a)$
\end{tabular}

4-fold WP and a 2 -fold WP, giving six atoms per unit cell. ${ }^{42} \mathrm{We}$ have checked that for binary compounds with different atomic species at each WP, the possible SG does not change, being the same as for monatomic pentamaterials. We present all possible SG supporting a monolayer pentagonal structure composed of one or two elements in Table 1.

This procedure can be extended to ternary penta-monolayer compounds. This is done by either replacing one atom in the 2 -fold WP or by replacing two atoms of the same type in the 4-fold WP (replacing one atom is also possible, but it generally gives the trivial space group $P 1$ as a result). The list of SG for ternary monolayer structures is given in Table 2 , obtained by choosing one SG in Table 1 and replacing the atoms in the WP as described, which yields a new SG with lower symmetry. Further increasing the number of elements is not pursued here since, in general, it will give similar low-symmetry SG as the ones listed in Tables 1 and 2.

Another route to explore is the stacking of pentagonal layers. We have studied translational (the so-called slip configurations, no rotation implied) stackings of few-layer pentagonal structures. The translational vectors were selected to comprise typical stackings such as $\mathrm{AA}, \mathrm{AB}, \mathrm{AC}$ and other, more exotic stackings. These stackings are amenable to automated SG calculation; we have obtained the SG for binary structures for two and three layers (composed of the same monolayer). The results are presented in the ESI. $\dagger$

Let us remark two features related to the obtained SG.

(i) There are planar and non-planar structures, which provide a means to study the effects of buckling in the electronic properties. For example, SG \#127 and \#55 can describe planar (atomically thin) structures, ${ }^{43}$ but they can also correspond to non-planar pentagonal structures with eight-coordinated atoms where an eight-fold WP is used to describe the site symmetry, such as in ref. 44 .

(ii) Restricting ourselves to monatomic and binary systems, SG\#113, SG\#100, SG\#90, SG\#32, SG\#18 and SG\#8 are noncentrosymmetric. This is one of the conditions needed for the appearance of nontrivial degeneracy points and lines in the electronic structure ${ }^{45}$ (see part 2). These noncentrosymmetric SG can also be subdivided in chiral and achiral groups depending on the presence of mirror and roto-inversion sym- 
Table 2 Ternary space groups supporting the pentagonal structure, arising from site substitutions in the SG of Table 1. Information retrieved from the Bilbao Crystallographic Server ${ }^{47}$

\begin{tabular}{llll}
\hline Parent group & WP change & Ternary group & Point group \\
\hline$\# 127^{a}$ & $2 \mathrm{a} \rightarrow 1 \mathrm{a}, 1 \mathrm{~b}$ & $P 4 / m(\# 83)$ & $4 / m$ \\
$\# 127$ & $4 \mathrm{~g} \rightarrow 2 \mathrm{i}, 2 \mathrm{~g}$ & $C m m m(\# 65)$ & $m m m$ \\
$\# 127$ & $4 \mathrm{~g} \rightarrow 2 \mathrm{a}, 2 \mathrm{a}$ & $P m c 2_{1}(\# 26)$ & $m m 2$ \\
$\# 113$ & $2 \mathrm{~b} \rightarrow 1 \mathrm{a}, 1 \mathrm{~b}$ & $P \overline{4}(\# 81)$ & $\overline{4}$ \\
$\# 113$ & $4 \mathrm{e} \rightarrow 2 \mathrm{e}, 2 \mathrm{~d}$ & $C m m 2(\# 35)$ & $m m 2$ \\
$\# 113$ & $4 \mathrm{e} \rightarrow 2 \mathrm{a}, 2 \mathrm{a}$ & $P 2_{1}(\# 4)$ & 2 \\
$\# 100$ & $2 \mathrm{a} \rightarrow 1 \mathrm{a}, 1 \mathrm{~b}$ & $P 4(\# 75)$ & 4 \\
$\# 100$ & $4 \mathrm{c} \rightarrow 2 \mathrm{e}, 2 \mathrm{~d}$ & $C m m 2(\# 35)$ & $m m 2$ \\
$\# 100$ & $4 \mathrm{c} \rightarrow 2 \mathrm{a}, 2 \mathrm{a}$ & $P c(\# 7)$ & $m$ \\
$\# 90$ & $2 \mathrm{c} \rightarrow 1 \mathrm{a}, 1 \mathrm{~b}$ & $P 4(\# 75)$ & 4 \\
$\# 90$ & $4 \mathrm{~d} \rightarrow 2 \mathrm{e}, 2 \mathrm{~g}$ & $C 222(\# 21)$ & 222 \\
$\# 90$ & $4 \mathrm{~d} \rightarrow 2 \mathrm{a}, 2 \mathrm{a}$ & $P 2_{1}(\# 4)$ & 2 \\
$\# 55$ & $2 \mathrm{a} \rightarrow 1 \mathrm{a}, 1 \mathrm{e}$ & $P 2 / m(\# 10)$ & $2 / m$ \\
$\# 55$ & $4 \mathrm{e} \rightarrow 2 \mathrm{~m}, 2 \mathrm{n}$ & $P 2 / m(\# 10)$ & $2 / m$ \\
$\# 55$ & $4 \mathrm{e} \rightarrow 2 \mathrm{a}, 2 \mathrm{~b}$ & $P m c 2(\# 26)$ & 2 \\
$\# 32$ & $2 \mathrm{a} \rightarrow 1 \mathrm{a}, 1 \mathrm{a}$ & $P 2(\# 3)$ & 2 \\
$\# 32$ & $4 \mathrm{c} \rightarrow 2 \mathrm{e}, 2 \mathrm{e}$ & $P 2(\# 3)$ & 2 \\
$\# 32$ & $4 \mathrm{c} \rightarrow 2 \mathrm{a}, 2 \mathrm{a}$ & $P c(\# 7)$ & $m$ \\
$\# 18$ & $2 \mathrm{a} \rightarrow 1 \mathrm{a}, 1 \mathrm{a}$ & $P 2(\# 3)$ & 2 \\
$\# 18$ & $4 \mathrm{~d} \rightarrow 2 \mathrm{e}, 2 \mathrm{e}$ & $P 2(\# 3)$ & 2 \\
$\# 18$ & $4 \mathrm{~d} \rightarrow 2 \mathrm{a}, 2 \mathrm{e}$ & $P 2(\# 4)$ & 2 \\
$\# 14$ & $2 \mathrm{a} \rightarrow 1 \mathrm{a}, 1 \mathrm{a}$ & $P \overline{1}(\# 2)$ & $\overline{1}$ \\
$\# 14$ & $4 \mathrm{e} \rightarrow 2 \mathrm{a}, 2 \mathrm{a}$ & $P 2_{1}(\# 4)$ & 2 \\
$\# 14$ & $4 \mathrm{e} \rightarrow 2 \mathrm{a}, 2 \mathrm{a}$ & $P c(\# 7)$ & 2 \\
$\# 8$ & $2 \mathrm{a} \rightarrow 1 \mathrm{a}, 1 \mathrm{~b}$ & $P m(\# 6)$ & $m$ \\
$\# 8$ & $4 \mathrm{~b} \rightarrow 2 \mathrm{a}, 2 \mathrm{a}$ & $P 1(\# 1)$ & 1 \\
$\# 8$ & $4 \mathrm{~b} \rightarrow 2 \mathrm{a}, 2 \mathrm{~b}$ & $P m(\# 6)$ & $m$ \\
& & & \\
& & &
\end{tabular}

${ }^{a}$ The SG \#117 gives the same ternary groups as the same WP coordinates are used for both groups.

metry operations. ${ }^{46}$ Thus, SG\#90 and SG\#18 are chiral and SG\#113, SG\#100, SG\#32 and SG\#8 are achiral.

We center the upcoming discussion in the noncentrosymmetric SG, as we aim to find novel examples of pentagonal systems with Weyl points in their electronic band structure. In the following we discuss the nature of degeneracy points in the band structure for some specific materials, based on the characteristics of their SG.

\subsection{Electronic structure of noncentrosymmetric pentagonal materials}

Since the proposal of penta-graphene, several pentagonal materials have been put forward. Most of the materials with one and two elements belong to SG \#14, \#113 and \#127. To our knowledge, there are not reported materials for the rest of the groups in Table 1. Those from SG \#14 are the most numerous; several databases for $2 \mathrm{D}$ materials report them. ${ }^{8,9}$ In fact, $\mathrm{PdSe}_{2}$, the only pentagonal 2D material synthesized to date, pertains to this SG. ${ }^{12}$ From these three groups, only SG \#113 is noncentrosymmetric. We focus on materials with noncentrosymmetric SG because they can host nodal points or lines, Weyl points and non-trivial topological features when SOC is relevant. In order to find more examples of those, we propose two possible routes. First, we search for ternary materials with noncentrosymmetric SG (Table 2). This is the case of SG \#4; a list of ternary materials has already been presented in ref. 48 .
We take an alternative route for SG \#4 and start with highly stable SG \#14 materials ${ }^{8}$ to derive new ternary materials via atomic substitution, following the information of Table 2. We propose two systems, PdSeS and PdSeTe, which are formed from $\mathrm{PdSe}_{2}$.

Another example is SG \#81, for which dynamically stable materials with formula $\mathrm{XP}_{5}(\mathrm{X}=\mathrm{Al}, \mathrm{Ga}, \mathrm{In})$ have also been proposed. ${ }^{20}$ Note that although $\mathrm{XP}_{5}$ materials are compositionally binary, from the group-theoretical viewpoint it can be considered ternary, given that one $\mathrm{P}$ atom is located in a WP different to the other four $\mathrm{P}$ atoms, breaking the two-glide plane symmetries present in the parent SG \# 113. We select the $\mathrm{InP}_{5}$ system for SG \#81; although it was studied previously, SOC was not considered. ${ }^{20}$ We include this effect in order to explore the spin texture and the appearance of Weyl points.

A second possible route is related to bilayers. Specifically, bilayers composed of SG \#113 monolayers with AB stacking give rise to noncentrosymmetric achiral SG \#111. However, we do not follow this approach in the present work.

To summarize, we have chosen three noncentrosymmetric SG for pentagonal 2D materials, namely, \#4, \#81, and \#113. We provide examples of the lattice structure for SG \#4 and \#81 in Fig. 2(a) and (c), respectively. Notice that the SG \#113 lattice is similar to the $\mathrm{SG} \# 81$, but the 4-coordinated atoms are equal for the \#113 case. Our selection for these particular SG is guided by two factors: on one hand, their proximity to recently reported materials, such as $\mathrm{PdSe}_{2}$, and on the other hand, the attractive chiral/achiral relationship of their nodal points and lines, as discussed below.

The first example we present is from SG \#4 $\left(P 2_{1}\right)$, with formula PdSeTe, which has been derived by atomic substitution of two Se atoms by Te atoms at a 4 -fold WP. The electronic band structure including SOC for PdSeTe is presented in Fig. 3(a), where the typical stick-together bands along the high symmetry line $\mathrm{X}-\mathrm{M}$ can be observed, due to the nonsym-

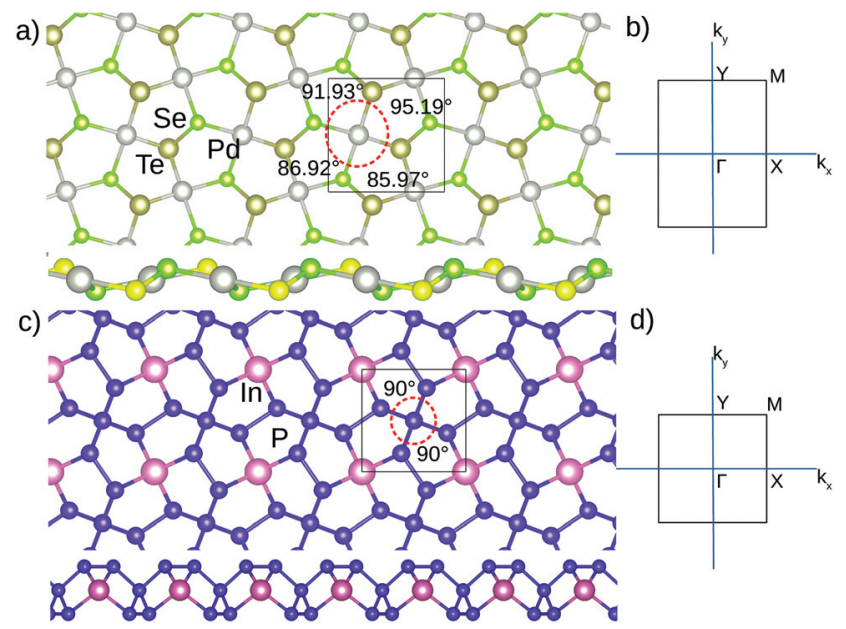

Fig. 2 Lattice structure for (a) SG \#4 and (c) SG \#81. Brillouin zone for (b) SG \#4 and (d) SG \#81. The angles given are measured considering only the planar projection and not the entire three-dimensional lattice structure. 
a)

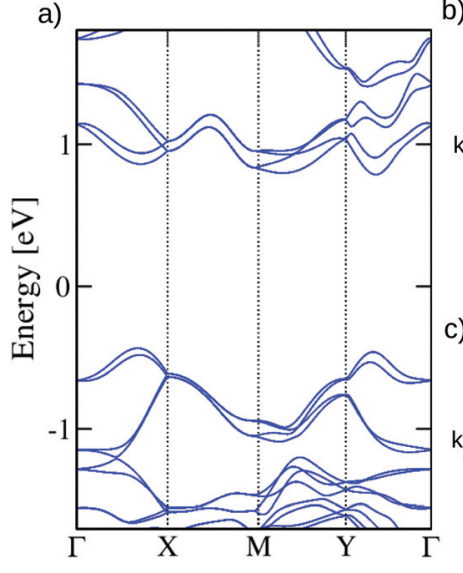

b)

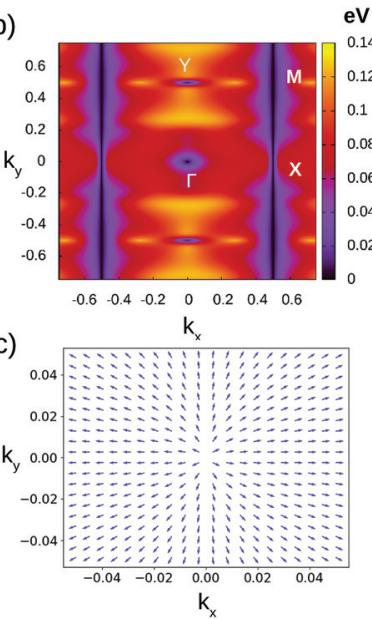

Fig. 3 (a) First-principles electronic band structure for PdSeTe. (b) A color map for the energy difference between the lowest pair of conduction bands mapped over the entire BZ for PdSeTe. (c) Spin texture for the fourth valence band in the vicinity of the $\Gamma$ point for PdSeTe.

morphic character of the SG. ${ }^{49}$ Further, around the $\Gamma$ point we can observe the spin splitting of bands, which show a 2 -fold degeneracy just at $\Gamma$.

In order to analyze the character of this nodal point we have numerically calculated the Berry phase $\mathrm{e}^{50}$ along a circular path around the $\Gamma$ point, which yields a value of $\pi$, indicating that it corresponds to a Weyl point with topological charge $|C|$ $=1 .^{51,52}$ This procedure is analogous to that performed for Weyl points in the three-dimensional case. ${ }^{53}$ Additionally, we present in Fig. 3(c) the spin texture of the fourth uppermost valence band to gain further insight into the nature of the Weyl node. For this particular band the spin texture shows a radial pattern near $\Gamma$, suggesting that these Weyl points are indeed a two-dimensional version of Kramers-Weyl points, as it was recently realized in other systems. ${ }^{45}$ Kramers-Weyl points are present in every chiral SG. They are pinned to highsymmetry points as long as no nodal lines are connected to them. ${ }^{54}$ In view of this, the Y point at the corner of the $\mathrm{BZ}$ can also host a Kramers-Weyl node, as it can been seen from Fig. 3(a). From a direct analysis of the physically irreducible representations (irreps) of this $\mathrm{SG}^{40}{ }^{40}$ it can be seen that the $\Gamma$ and Y points only have two-dimensional irreps, indicating that only Weyl nodes can be formed. Note that only two dimensional irreps are possible along the $\mathrm{X}-\mathrm{M}$ high-symmetry line, thus implying the formation of a Weyl nodal line.

This nodal line also has a little group that only has a twodimensional irrep if time-reversal holds. This irrep can be expressed as $e^{i \pi u} i \sigma_{z}$, where $u$ is the fractional coordinate along the nodal line. ${ }^{40}$ Following the results in ref. 55 and 56, the ratio $\alpha_{v} / \alpha_{\mu}$, where $\alpha_{v / \mu}$ are the irrep eigenvalues, indicates the type of Weyl point present along a symmetry line. In this case $\alpha_{\mathrm{v}} / \alpha_{\mu}=-1$, meaning that a symmetry-protected Weyl node is produced at every point along the BZ boundary, with Chern number of magnitude $|C|=1$.
These features should be present in every material with the SG \#4 and time-reversal symmetry. These point nodes and nodal lines are protected by the combined action of time-reversal $T$ and $2_{1}$ symmetries $\left(T C_{2} \mid 1 / 2,0,0\right) .{ }^{41,57}$ Similar results are obtained for PdSeS, which are presented in the ESI. $\uparrow$ The Weyl points and nodal line can be clearly identified in a color map plot of the energy difference between two adjacent bands, as shown in Fig. 3(b), for the lowest pair of conduction bands, where the Weyl nodes are clearly spotted and the nodal line appears along all the $\mathrm{X}-\mathrm{M}$ line.

Next, we turn to the SG $\# 81(P \overline{4})$, presenting the first-principles band structure with SOC for $\operatorname{InP}_{5}$ in Fig. 4(a). The spin splitting can also be observed, being larger for the lowest conduction bands near the $\Gamma$ point. We also have computed the Berry phase for this material, obtaining a value of $\pi$ for all the high-symmetry points of interest, mentioned below. In this case the lattice structure is achiral, due to the presence of rotoinversion $\mathrm{S}_{4}$ and mirror planes. Still, as mentioned in ref. 45, there exists the possibility to have a reminiscence of chirality, so to say, at momentum space. This is because certain little groups (at high-symmetry points) of these achiral groups could be chiral. ${ }^{47}$ This is the case for SG \#81, where the $\mathrm{X}(\mathrm{Y})$ point located at $[1 / 2,0]([0,1 / 2])$ has a chiral little group. ${ }^{41}$ To explore this assertion, the spin texture for the first conduction band is presented in Fig. 4(c), in the vicinity of the X point. It can be observed that the radial pattern is partially present, which is related to the competition of the spin-orbit interactions that can be present in the neighborhood of $\mathrm{X}$, due to the $C_{2}$ little group. This competition distorts the spin texture and masks the chiral signatures of the node.

It can be mentioned that at the $\Gamma$ point (and also at the $\mathrm{M}$ point) there exists a Weyl point that is hosted by an achiral little group, and therefore no Kramers-Weyl node is allowed. ${ }^{58}$
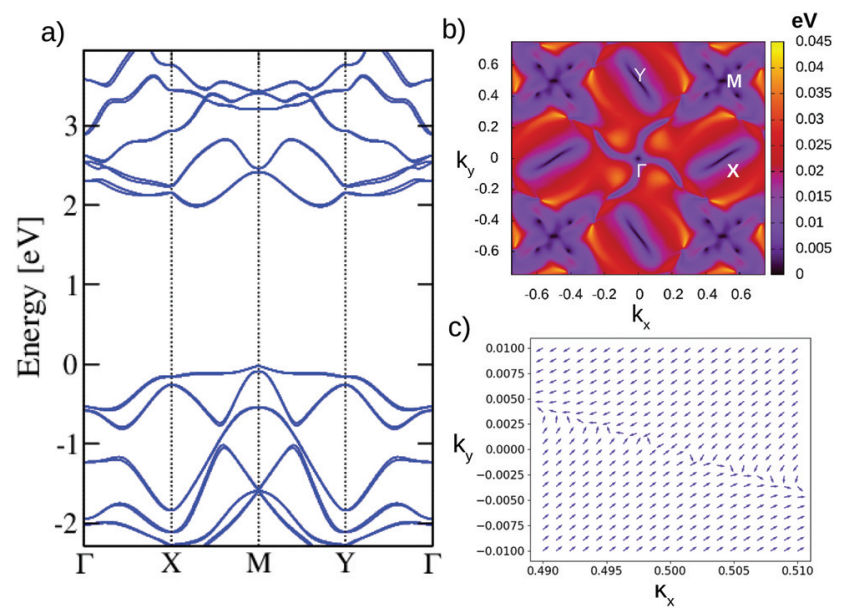

Fig. 4 (a) Electronic band structure for $\operatorname{lnP}_{5}$. (b) Color map for the energy difference between the third and fourth uppermost valence bands, mapped over the entire $\mathrm{BZ}$ for $\mathrm{InP}_{5}$. Darker zones away from high-symmetry points are not degenerate, but very close in energy. (c) The spin texture for the first conduction band for a region near the $\mathrm{X}$ point for $\ln P_{5}$. 
One of the interesting characteristics of these points $(\Gamma$ and $M)$ is that they can be considered as two-dimensional projections of Kramers-Weyl nodal lines. ${ }^{54}$ That is to say, they correspond separately to the end points of two nodal lines that would exist in a three-dimensional BZ for the same SG along $\Gamma-\mathrm{A}$ and $\mathrm{M}-$ $\mathrm{R}^{54}$ Notice also that there are no nodal lines, since only onedimensional irreps are present away from the high-symmetry points. ${ }^{47}$ All these facts can be better appreciated in the color map of the spin splitting (energy difference) for the second pair of adjacent valence bands in Fig. 4(b). It can be seen that the Weyl nodes appear exactly at the high-symmetry points, along with zones of relative high and low spin splitting. Similar conclusions could be attained for the SG \#111 $(P \overline{4} 2 m)$, where the $\mathrm{X}(\mathrm{Y})$ point also hosts Kramers-Weyl nodes. The reason is the same as for SG \#81. As was mentioned, this SG can be formed by a bilayer composed of two (equal) SG \#113 monolayers with AB stacking. We leave the detailed analysis of multilayer pentagonal structures for a later work.

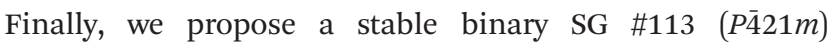
material, $\mathrm{GeBi}_{2}$. Its band structure with SOC is presented in Fig. 5(a). In this achiral SG, the M point little group protects a fourfold degenerate node. ${ }^{59,60}$ This can be clearly observed in the band structure of Fig. 5, as for example in the upper group of valence bands, where the fourfold fermion locates at an energy of $-0.20 \mathrm{eV}$. This fermion is not chiral, since the little group of the $\mathrm{M}$ point is isomorphic to $D_{2 \mathrm{~d}} \cdot{ }^{47}$ The rest of the high-symmetry points have two-fold Weyl points with no chiral character, namely, the $\Gamma, \mathrm{X}$ and $\mathrm{Y}$ points. However, only the $\Gamma$ point comprises an isolated node, since the $\mathrm{X}$ and $\mathrm{Y}$ points are part of a closed twofold nodal line that goes around the whole BZ boundary, as was reported in our previous work. ${ }^{42}$ The analysis for this nodal line is analogous to that of the SG \#4 case. The ratio for the $2 \mathrm{D}$ irrep in the little group is $\alpha_{\mathrm{v}} / \alpha_{\mu}=-1$, giving again a line of Weyl points all along the BZ boundary, with $|C|=1 .^{56}$ We should mention that this twofold nodal line
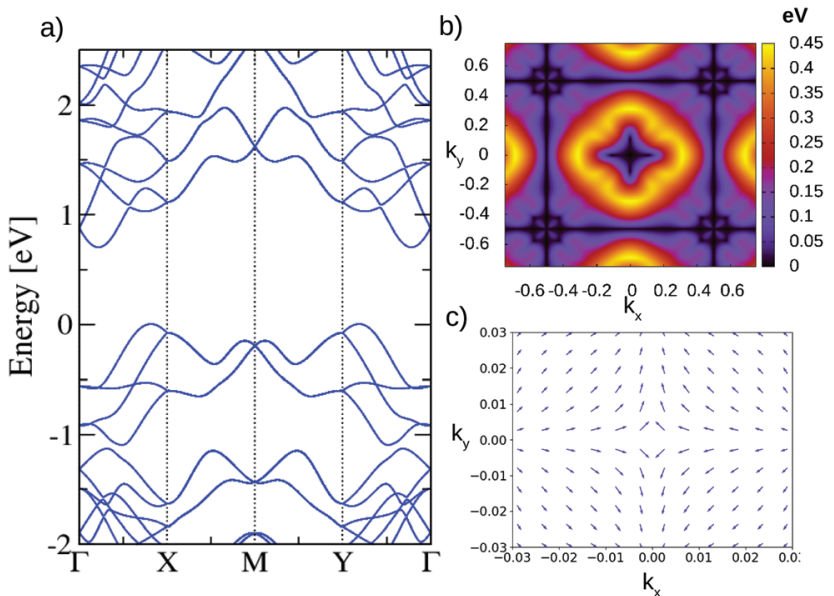

Fig. 5 (a) First-principles electronic band structure for $\mathrm{GeBi}_{2}$. (b) Color map for the energy difference between the two uppermost valence bands mapped over the entire $B Z$ for $G_{e B i}$. (c) Spin texture for the uppermost valence band near the $\Gamma$ point for $\mathrm{GeBi}_{2}$. is formed by the splitting of a fourfold nodal line present in the case without SOC. The two-dimensional character of this nodal line, and that occurring in SG \#4, is an interesting feature that has attracted the attention of several groups. ${ }^{61-65}$

To recapitulate, in this SG we encounter the peculiar coexistence of a fourfold fermion, a twofold Weyl node and a closed twofold nodal line, which gives several possibilities to study the interaction between degenerate fermions. Additional insight can be acquired by looking at Fig. 5(b), where all the nodal points and the closed nodal line mentioned above are clearly shown in the color map for the upper pair of valence bands. We complete this characterization by presenting the spin texture for the top valence band in the vicinity of the $\Gamma$ point in Fig. 5(c). It shows a Dresselhaus-like spin texture, as allowed by the point group associated to SG \#113. ${ }^{41}$ The fourfold $\mathrm{M}$ point also presents this type of spin-orbit texture, as it can be observed in Fig. S.21 in the ESI. $\dagger$

The diverse patterns arising in the spin textures can be better explained by resorting to an effective model that we present in the Appendix. There, it is shown that the spin texture patterns depend on the relative magnitude of the coefficients accompanying the momentum components in the $k \cdot p$ Hamiltonian. One of the most appealing features of the SG \#4 materials is that the spin texture depends not only on the spin-orbit interaction but also on the anisotropy due to the low spatial symmetry. This is illustrated in Fig. 3(c) and, more conspicuously, in Fig. 4(c). Likewise, the distortion of the spin texture due to the competition between SOC and anisotropy found in group \#81 is further analyzed in the Appendix. Different spin textures are realized for each band in these materials; several examples are depicted in the Appendix illustrating the situations found in the three groups under study. It is important to note that the combination of anisotropy and nontrivial symmetry may produce different spin textures in nodes belonging to the same material. ${ }^{66}$

We have chosen several penta-materials to illustrate the characteristics associated with different SG. We would like to emphasize that, to the best of our knowledge, there are not any reported materials for the SG $\# 90$ to this date. Nonetheless, this group is of great interest, since it can host chiral multifold fermions. ${ }^{67}$ In particular, at the $\mathrm{M}$ point this SG has a chiral fourfold degenerate fermion, with a double spin-1/2 representation. ${ }^{59,67}$ Physically, it can be viewed as two copies of twofold Weyl fermions, each one with a Berry phase of $\pi$. Therefore, their Chern numbers verify $|C|=1$. Also a twofold chiral fermion is present at $\Gamma$, and twofold nodal lines along the whole BZ boundary are present, similar to the SG \#113 but with chiral nature. This implies that the interplay between chiral multifold and chiral twofold fermions could be studied in this particular SG. Finding a material realization of this SG would certainly be appealing.

\subsection{Optical properties}

The existence of Weyl points is related to enhanced physical responses, compared to materials without topological nodes. For example, a large magnetoresistance ${ }^{68,69}$ in magnetic 
materials, transport characteristics such as the chiral magnetic effect, the nonlinear anomalous Hall effect, the kinetic Faraday effect and the gyrotropic magnetic effect, also called kinetic magnetoelectric effect. ${ }^{51,70,71}$ Notice that Weyl points are not always at the Fermi level, so electrostatic gating or doping might be needed. ${ }^{72,73}$ In gapped materials, as these presented here, optical properties are the straightforward method to probe their features without further tuning. In addition, optical effects are among the most promising responses due to the momentum space selection rules, that could give characteristic features at certain frequencies. ${ }^{45,74}$ Here we report optical first-principles calculations in the Wannier basis for the materials previously analyzed. Specifically, we have computed the optical conductivity, the rotatory power in terms of the natural optical activity (NOA) and the interband contribution to the nonlinear shift current (for details see section 2). In what follows we describe some of the main aspects of the optical properties.

First, all calculations of the optical properties were carried out using Wannier-interpolated models, as commented before. The specific orbitals included depend on the material; they were detailed in section 2 . These choices yield a good agreement for the low-energy bands. The Wannier band structures for each material are presented in the ESI. $\dagger$

We begin by reporting the optical conductivity for PdSeTe, projected in the spin $z$ direction, which is presented in Fig. 6 . It can be seen that no spin polarization is present in this system (other spin directions give similar results). Due to the low symmetry of this SG, there are nonzero off-diagonal (Halllike) components of the optical conductivity, which are two orders of magnitude smaller than the longitudinal part. Still, this could give rise to photoinduced currents in the transverse directions and also to optical rotation. The low symmetry also implies that there are few selection rules for interband transitions in momentum space. In consequence, contributions from all the $\mathrm{BZ}$ make the distinction of peaks rather opaque.
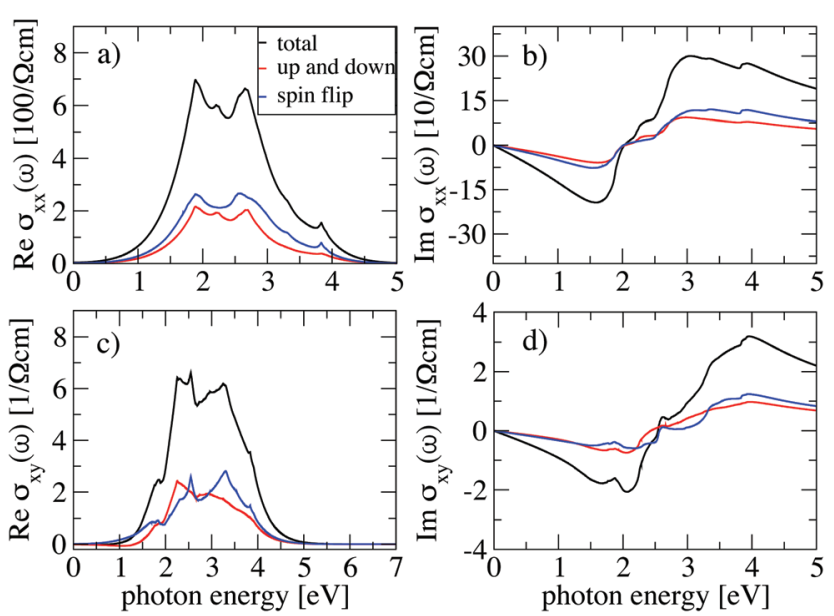

Fig. 6 Optical conductivity for PdSeTe with spin components along the $z$ direction. (a) Real part for $\sigma_{x x}$ (b) Imaginary part of $\sigma_{x x}$. (c) Real part for $\sigma_{x y \cdot}(\mathrm{d})$ Imaginary part of $\sigma_{x y}$.
In order to gain insight about the optical response we calculated the interband contribution to the rotatory power, obtained from the NOA tensor, as shown in Fig. 7(a) for PdSeTe. There exists a nonzero response in a wide range of frequencies, which gives to this material a great potential for optoelectronic applications. ${ }^{75}$ We hypothesize that such response could be augmented by using multilayers, since the chiral nature is enhanced by increasing thickness. ${ }^{76}$ Even a higher outcome could be obtained if rotated layers are employed, as previous works on twisted materials have reported. ${ }^{77,78}$ Note that the orbital contribution is generally greater than the spin contribution, with the exception of the low-frequency region, including the zone below the absorbing edge $(\sim 1.2[\mathrm{eV}])$. This is shown in Fig. 7(b), where it is evident that the spin contribution is of the same order as the orbital contribution, even surpassing it close to the gap. This last behavior is encountered also in the NOA response of $\mathrm{GeBi}_{2}$ along the whole frequency range, as shown in Fig. S.23 in the ESI. $\dagger$ This is in fair contrast with other materials such as elemental tellurium, where the spin contribution was found to be marginal. ${ }^{71}$

Nonlinear optical effects occurring in these materials with chiral nodes have special relevance for potential applications. $^{79-82}$ In order to explore this issue, we computed the interband contribution to the nonlinear shift current $\sigma_{\mathrm{abc}}$, which arises due to the charge center shift associated to the effects of a nonzero interband Berry connection. ${ }^{79,83}$ The effect depends on the type of (linear) polarization of light and the symmetry character of the $\sigma_{\mathrm{abc}}$ tensor, given in eqn (4). For PdSeTe(S) (SG \#4) the tensor has 4 independent nonzero components, while for $\operatorname{InP}_{5}$ (SG \#81) the tensor has 2 nonzero independent components. ${ }^{84}$ For the purpose of yielding more reliable results, the shift current of the layered system is rescaled by a numerical factor taking into account the slab geometry, as implemented in ref. 74 and 85 . This factor is defined as the ratio between the length of the vacuum region
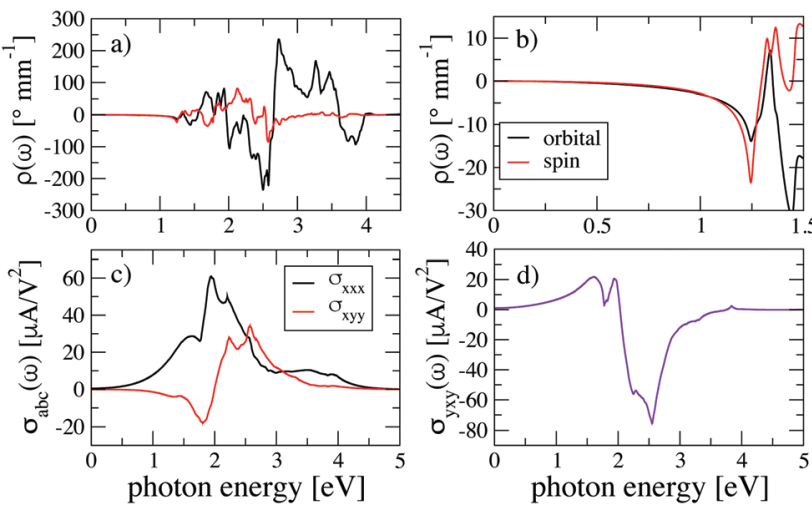

Fig. 7 (a) Rotatory power from NOA for PdSeTe with orbital and spin contribution. (b) Zoom-in from (a) along the region below the optical direct bandgap. (c) Shift current along the $x$ direction with in-plane polarization for PdSeTe. (d) Shift current along the $y$ direction with inplane polarization for PdSeTe. 
$d_{\mathrm{vac}}$ and the monolayer thickness $d_{\mathrm{m}}$, such that the final shift current tensor $\sigma_{\mathrm{abc}}$ is given by ${ }^{74}$

$$
\sigma_{\mathrm{abc}}=\left(\frac{d_{\mathrm{vac}}}{d_{\mathrm{m}}}\right) \sigma_{\mathrm{abc}}^{\text {layer }}
$$

We present the shift current tensor for PdSeTe along the $x$ and $y$ directions in Fig. $7(\mathrm{c})$ and (d). The rest of the components are not shown because they have a negligible value in comparison. Additionally, in Fig. 8 the shift current for $\mathrm{InP}_{5}$ is shown, with values in other polarization directions, specifically, in the $z$ direction.

This current is more sensitive to specific regions in momentum space, at variance with other optical responses. ${ }^{86}$ Peaks in these currents are slightly related to peaks in the optical conductivity contributions since two-band and three-band transitions are the most important weights. ${ }^{59,86,87}$ Points where Weyl nodes are located ( $\Gamma$ and $\mathrm{Y}$ ) have a large contribution, as for example in $\sigma_{x y y}$ for PdSeTe, depicted in Fig. 7(c). The peaks are localized within regions which coincide with the energy difference between two Weyl points. Unfortunately, this contribution from Weyl points is not exclusive for this particular energy range, due to the low symmetry of the material and to the proximity in energy of trivial bands along the BZ.

To sum up, whereas Weyl points contribute significantly to the shift current and, in fact, certain peaks in the spectrum can be attributed to them, no intrinsic Weyl signatures are expected in this system, such as it occurs in the circular photogalvanic effect. ${ }^{86}$ A similar conclusion is reached by inspecting the shift current of $\mathrm{InP}_{5}$ in Fig. 8, where achiral Weyl nodes do not show any particular features. Still, there are certain aspects that can be pointed out, such as the occurrence of anisotropy, which is observed by contrasting different components of the $\sigma_{y x y}$ tensor. Since the contribution to the shift current from these components depends on the polarization of light, anisotropy introduces selectivity along some particular spatial directions. This is highly desirable for applications in photovoltaics, which requires preferred directions in materials in order to efficiently transport the induced current to the electrodes. ${ }^{83}$

One of the main drawbacks of this bulk photovoltaic response is the low conversion ratio, which can hinder the applicability of the effect. ${ }^{79}$ Consequently, the magnitude of the shift current is also important. ${ }^{88,89}$ The search for novel materials with high shift currents is thus the focus of great interest. ${ }^{80}$ In this line, we would like to emphasize the remarkable values obtained for the pentagonal material PdSeTe for
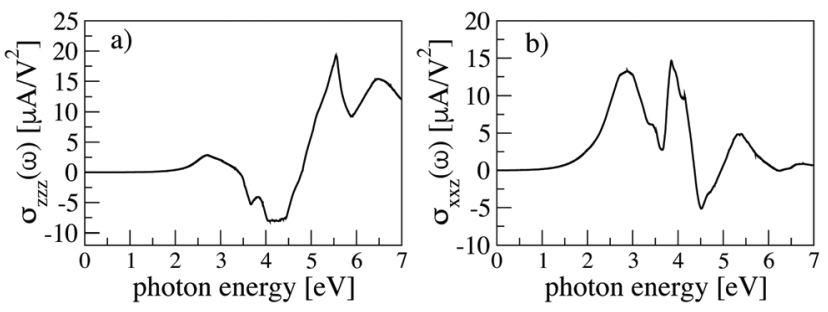

Fig. 8 Components of the shift current tensor $\sigma_{\mathrm{abc}}(\omega)$ for $\ln \mathrm{P}_{5}$. the component $\sigma_{x x x}$, as shown in Fig. 7(c), with a peak value of $60\left[\mu \mathrm{A} \mathrm{V}^{-2}\right]$ at $\sim 1.9 \mathrm{eV}$ and for $\sigma_{y x y}$ in Fig. $7(\mathrm{~d})$, with a peak value of $-76\left[\mu \mathrm{A} \mathrm{V}^{-2}\right]$ at $\sim 2.5 \mathrm{eV}$. We can also observe in Fig. 8(a) that the $\sigma_{z z z}$ component in In $\mathrm{P}_{5}$ has a peak value of $130\left[\mu \mathrm{A} \mathrm{V}^{-2}\right]$ at $\sim 5.55 \mathrm{eV}$. Finally, $\sigma_{x x z}$ has two peaks of $89\left[\mu \mathrm{A} \mathrm{V}^{-2}\right]$ and $98\left[\mu \mathrm{A} \mathrm{V}^{-2}\right]$ at $\sim 2.88 \mathrm{eV}$ and $\sim 3.87 \mathrm{eV}$, respectively. These magnitudes compare very favorably with previously reported values for layered materials, as for example with monolayer GeS, which possesses a shift current of $100\left[\mu \mathrm{A} \mathrm{V}^{-2}\right] .{ }^{85}$ Furthermore, since the values are within the visible/UV spectrum, they convey very promising prospects for these systems as photovoltaic materials. ${ }^{90}$

As it is well-known, the use of the PBE functional underestimates the band gaps. This also has an obvious consequence in the optical spectra, producing a redshift of the optical features. For those materials including a transition group element, we also calculated the electronic properties with a hybrid functional, showing that our results are not affected by this approximation, besides the expected energy displacement mentioned above.

In the $\mathrm{ESI}_{\dagger} \dagger$ we present additional material related to the optical response of $\mathrm{InP}_{5}$ and for $\mathrm{GeBi}_{2}$ (SG \#113).

\section{Conclusions}

In this work we have classified all the layer groups that can support a pentagonal structure with up to three elements. We have also classified the multilayer structures with slip stackings up to three layers. We have focused on specific noncentrosymmetric SG, with chiral and achiral point groups. The corresponding SG were described in detail and their degeneracies linked to Weyl nodes and Kramers-Weyl nodes depending on their little group. This framework was subsequently applied to several examples of pentagonal materials to describe their electronic structures. Additionally, their optical properties were reported, complementing the description of their electronic band structures. Although the existence of Weyl points in these materials does not imply any specific signature in their physical properties, we have shown that they can be related to the enhancement of the shift currents, yielding high values of this magnitude.

We expect that pentagonal materials such as PdSeTe and PdSeS to be fairly feasible experimentally, since they are closely related with the recently synthesized pentagonal material $\mathrm{PdSe}_{2}$. Other routes that are worth to be explored further are the signatures of the spin textures and their spinorbit competition and anisotropy. Also, multilayers are highly attractive since little work has been done for pentagonal systems and, as we mentioned above, different stackings could give emergent SG with contrasting symmetry-related properties or boost chirality-related phenomena. Finally, our results indicate that the optical response for other pentagonal materials deserves to be explored, since the interplay of Weyl nodes, lowsymmetry and chemical composition could yield novel systems with enhanced photovoltaic currents. 


\section{Conflicts of interest}

There are no conflicts to declare.

\section{Appendix: effective models and spin textures for two-fold Weyl points}

Since the degenerate points are local properties in momentum space, effective models are helpful to understand in more detail the relation between structural chirality and spin textures. Here we analyze specific nodal points in SG \#4, SG \#81 and SG \#113, selected from those presented in section 3.

\section{SG \#4 and SG \#81}

For both groups, the most interesting $k$ points are those with chiral little groups ( $\Gamma$ and $\mathrm{Y}$ for SG \#4 and X for SG \#81). These little groups are all isomorphic to $C_{2},{ }^{47}$ which has only one symmetry operation, namely, a $\pi$ rotation about the axis perpendicular to the layer plane. Using the theory of invariants, ${ }^{91}$ the most general $k \cdot p$ effective model can be expressed up to linear order in the crystal momentum

$$
H_{C_{2}}=\alpha_{0}+\alpha_{1 x x} k_{x} \sigma_{x}-\alpha_{1 x y} k_{x} \sigma_{y}+\alpha_{1 y x} k_{y} \sigma_{x}-\alpha_{1 y y} k_{y} \sigma_{y}
$$

where $k_{i}$ is the crystal momentum in the $i$ direction measured from the corresponding high-symmetry point, $\sigma_{i}$ are the Pauli matrices; $\alpha_{0}$ and $\alpha_{1 i j}$ correspond to the free parameters that describe the energy bands in the vicinity of the chiral point. Note that this model allows for the presence of anisotropy, given that different parameters are associated with the $x$ and $y$ momentum components. Besides, depending on the sign relation of these parameters, different spin-orbit interactions can be realized. Thus, a competition between spin-orbit effects could be present, depending on the relative magnitudes and signs of the linear parameters. The competing interplay of both effects, anisotropy and spin-orbit, produces in some cases spin textures appreciably different from the ideal radial textures which are characteristic of Kramers-Weyl nodes. ${ }^{45}$ These different spin textures appear by choosing different values for the parameters $\alpha_{1 i j}$ in eqn (6).

For instance, to realize the spin textures that are observed in the pentagonal materials of SG \#4, an additional condition is imposed such that $\alpha_{1 x y} \ll \alpha_{1 x x} \alpha_{1 y y}$ and $\alpha_{1 y x} \ll \alpha_{1 x x} \alpha_{1 y y}$. Taking this into account, the Hamiltonian in eqn (6) can be reduced to

$$
H_{\mathrm{SG} \mathrm{\# 4}}=\alpha_{0}+\alpha_{1 x x} k_{x} \sigma_{x}-\alpha_{1 y y} k_{y} \sigma_{y}
$$

For the sake of illustrating this point, Fig. 9(a) and (b) depict these cases for parameter values chosen without the aim of modeling a particular material. In Fig. 9(a) a Dresselhaus-like texture is obtained by using the constraint $\operatorname{sign}\left(\alpha_{1 x y}\right)=\operatorname{sign}\left(\alpha_{1 x x}\right)$. This texture can be appreciated in the uppermost valence bands of PdSeTe as shown in Fig. S12 of the ESI. $\dagger$ In the case of Fig. 9(b) the condition $\operatorname{sign}\left(\alpha_{1 x y}\right)=$ $-\operatorname{sign}\left(\alpha_{1 x x}\right)$ gives a radial spin texture, very similar to the one
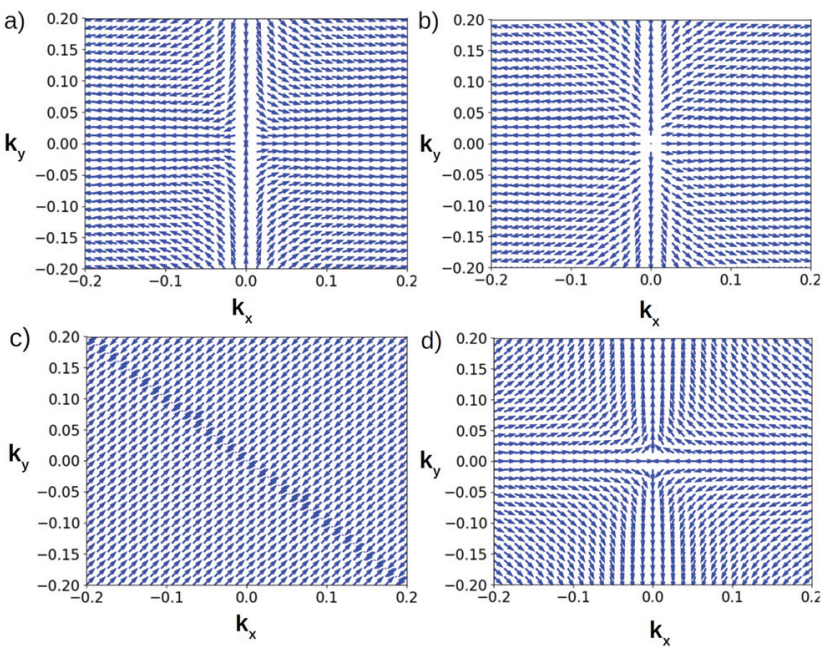

Fig. 9 Spin texture from the $k \cdot p$ model for (a) SG \#4 with $\operatorname{sign}\left(\alpha_{1 x y}\right)=$ $\operatorname{sign}\left(\alpha_{1 x x}\right)$ (b) SG \#4 with $\operatorname{sign}\left(\alpha_{1 x y}\right)=-\operatorname{sign}\left(\alpha_{1 x x}\right)$ (c) X point in SG \#81 and (d) $\Gamma$ point in SG \#113.

presented above for PdSeTe in Fig. 3(c). The competition and variability in spin textures within the same material generalizes the exclusive radial textures encountered in previous works and opens the way to explore the interplay of anisotropy and competing spin-orbit couplings in pentagonal systems.

For SG \#81 at the X point, the model must include all $\alpha_{1 i j}$ parameters, resulting in the interplay between all spin-orbit couplings, which yields diverse textures. In Fig. 9(c) a spin texture with a partial inward radial distribution is presented. This is in good agreement with the spin texture near $\mathrm{X}$, obtained for In $\mathrm{P}_{5}$ in Fig. 4(c).

\section{SG \#113}

For this SG the only nodal point that can be treated with a twoband model is the $\Gamma$ point, which has an achiral little group isomorphic to $D_{2 \mathrm{~d}}$. In this case, the effective model is isotropic, and up to linear order in crystal momentum is given by

$$
H_{\mathrm{SG} \mathrm{\# 113}}=\beta_{0}+\beta_{1}\left(k_{x} \sigma_{x}-k_{y} \sigma_{y}\right),
$$

where $\beta_{i}$ are free parameters. This model produces isotropic spin textures in the neighborhood of the $\Gamma$ point, as it can be observed in Fig. 9(d), where a Dresselhaus spin texture arises. This compares well with the results for $\mathrm{GeBi}_{2}$ depicted in Fig. 5(c), which indicates the dominating spin-orbit interaction is this material. The analysis of the fourfold fermion requires a four-band model along with new symmetry considerations, which are outside the scope of this Appendix.

\section{Acknowledgements}

This work has been partially supported by Chilean FONDECYT grant 1201876, Spanish MCIU and AEI and the European Union under grant no. PGC2018-097018-B-I00 (MCIU/AEI/ FEDER, UE), and by Universidad Técnica Federico Santa María 
grant USM-DGIIP PI-LI 1925 (Chile). V. N. acknowledges financial support from Beca Doctorado Nacional ANID no. 21170872 (CL). J. D. C. acknowledges Universidad de Medellín under grant 1120 and the Laboratorio de Simulación y Computación Científica for computational facilities.

\section{References}

1 P. Miró, M. Audiffred and T. Heine, Chem. Soc. Rev., 2014, 43, 6537-6554.

2 K. Novoselov, A. Mishchenko, A. Carvalho and A. H. Castro Neto, Science, 2016, 353, aac9439.

3 D. Saptarshi, J. A. Robinson, M. Dubey, H. Terrones and M. Terrones, Annu. Rev. Mater. Res., 2015, 45, 1-27.

4 G. Fiori, F. Bonaccorso, G. Iannaccone, T. Palacios, D. Neumaier, A. Seabaugh, S. K. Banerjee and L. Colombo, Nat. Nanotechnol., 2014, 9, 768-779.

5 Q. H. Wang, K. Kalantar-Zadeh, A. Kis, J. N. Coleman and M. S. Strano, Nat. Nanotechnol., 2012, 7, 699-712.

6 A. Jain, S. P. Ong, G. Hautier, W. Chen, W. D. Richards, S. Dacek, S. Cholia, D. Gunter, D. Skinner, G. Ceder and K. A. Persson, APL Mater., 2013, 1, 011002.

7 K. Choudhary, I. Kalish, R. Beams and F. Tavazza, Sci. Rep., 2017, 7, 5179.

8 S. Haastrup, M. Strange, M. Pandey, T. Deilmann, P. S. Schmidt, N. F. Hinsche, M. N. Gjerding, D. Torelli, P. M. Larsen, A. C. Riis-Jensen, J. Gath, K. W. Jacobsen, J. J. Mortensen, T. Olsen and K. S. Thygesen, 2D Mater., 2018, 5, 042002.

9 N. Mounet, M. Gibertini, P. Schwaller, D. Campi, A. Merkys, A. Marrazzo, T. Sohier, I. E. Castelli, A. Cepellotti, G. Pizzi and N. Marzari, Nat. Nanotechnol., 2018, 13, 246-252.

10 K. Choudhary, K. F. Garrity, A. C. E. Reid, B. DeCost, A. J. Biacchi, A. R. H. Walker, Z. Trautt, J. Hattrick-Simpers, A. G. Kusne, A. Centrone, A. Davydov, J. Jiang, R. Pachter, G. Cheon, E. Reed, A. Agrawal, X. Qian, V. Sharma, H. Zhuang, S. V. Kalinin, B. G. Sumpter, G. Pilania, P. Acar, S. Mandal, K. Haule, D. Vanderbilt, K. Rabe and F. Tavazza, npj Comput. Mater., 2020, 6, 173.

11 L. Talirz, S. Kumbhar, E. Passaro, A. V. Yakutovich, V. Granata, F. Gargiulo, M. Borelli, M. Uhrin, S. P. Huber, S. Zoupanos, C. S. Adorf, C. W. Andersen, O. Schütt, C. A. Pignedoli, D. Passerone, J. VandeVondele, T. C. Schulthess, B. Smit, G. Pizzi and N. Marzari, Sci. Data, 2020, 7, 299.

12 A. D. Oyedele, S. Yang, L. Liang, A. A. Puretzky, K. Wang, J. Zhang, P. Yu, P. R. Pudasaini, A. W. Ghosh, Z. Liu, C. M. Rouleau, B. G. Sumpter, M. F. Chisholm, W. Zhou, P. D. Rack, D. B. Geohegan and K. Xiao, J. Am. Chem. Soc., 2017, 139, 14090-14097.

13 C.-P. Tang, S.-J. Xiong, W.-J. Shi and J. Cao, J. Appl. Phys., 2014, 115, 113702.

14 S. Zhang, J. Zhou, Q. Wang, X. Chen, Y. Kawazoe and P. Jena, Proc. Natl. Acad. Sci. U. S. A., 2015, 112, 2372-2377.
15 T. Zhao, S. Zhang, Y. Guo and Q. Wang, Nanoscale, 2016, 8, 233-242.

16 Y. Ma, L. Kou, X. Li, Y. Dai and T. Heine, NPG Asia Mater., 2016, 8, e264.

17 J. Li, X. Fan, Y. Wei, H. Liu, S. Li, P. Zhao and G. Chen, Sci. Rep., 2016, 6, 33060.

18 L.-S. Zhao, Y. Wang, C.-P. Chen, L.-L. Liu, H.-X. Yu, Y. Zhang, Y. Chen and X.-C. Wang, Phys. E, 2017, 91, 82-87.

19 Q. Pang, L. Liu and J. Zhao, J. Appl. Phys., 2017, 122, 094302.

20 M. Naseri, Chem. Phys. Lett., 2018, 706, 99-106.

21 M. Naseri, S. Lin, J. Jalilian, J. Gu and Z. Chen, Front. Phys., 2018, 13, 138102.

22 M. Naseri, Phys. Lett. A, 2018, 382, 710-715.

23 L. Liu, I. Kankam and H. L. Zhuang, Phys. Rev. B, 2018, 98, 205425.

24 K. Zhao, Y. Guo, Y. Shen, Q. Wang, Y. Kawazoe and P. Jena, J. Phys. Chem. Lett., 2020, 11, 3501-3506.

25 H. Liu, G. Qin, Y. Lin and M. Hu, Nano Lett., 2016, 16, 3831-3842.

26 B. Xiao, Y.-c. Li, X.-f. Yu and J.-b. Cheng, ACS Appl. Mater. Interfaces, 2016, 8, 35342-35352.

27 S. Winczewski and J. Rybicki, Carbon, 2019, 146, 572-587.

28 A. D. Oyedele, S. Yang, T. Feng, A. V. Haglund, Y. Gu, A. A. Puretzky, D. Briggs, C. M. Rouleau, M. F. Chisholm, R. R. Unocic, D. Mandrus, H. M. Meyer, S. T. Pantelides, D. B. Geohegan and K. Xiao, J. Am. Chem. Soc., 2019, 141, 8928-8936.

29 G. D. Nguyen, A. D. Oyedele, A. Haglund, W. Ko, L. Liang, A. A. Puretzky, D. Mandrus, K. Xiao and A.-P. Li, ACS Nano, 2020, 14, 1951-1957.

30 L. Liu and H. L. Zhuang, Phys. Rev. Mater., 2018, 2, 114003. 31 H. L. Zhuang, Comput. Mater. Sci., 2019, 159, 448-453.

32 L. Liu, I. Kankam and H. L. Zhuang, Electron. Struct., 2019, 1, 015004.

33 M. Rao, 2017, arXiv:1708.00274, arXiv e-prints.

34 P. Giannozzi, O. Baseggio, P. Bonfà, D. Brunato, R. Car, I. Carnimeo, C. Cavazzoni, S. de Gironcoli, P. Delugas, F. Ferrari Ruffino, A. Ferretti, N. Marzari, I. Timrov, A. Urru and S. Baroni, J. Chem. Phys., 2020, 152, 154105.

35 J. Enkovaara, C. Rostgaard, J. J. Mortensen, J. Chen, M. Dułak, L. Ferrighi, J. Gavnholt, C. Glinsvad, V. Haikola, H. Hansen, et al., J. Phys.: Condens. Matter, 2010, 22, 253202.

36 A. A. Mostofi, J. R. Yates, G. Pizzi, Y.-S. Lee, I. Souza, D. Vanderbilt and N. Marzari, Comput. Phys. Commun., 2014, 185, 2309-2310.

37 Q. Wu, S. Zhang, H.-F. Song, M. Troyer and A. A. Soluyanov, Comput. Phys. Commun., 2018, 224, 405-416.

38 A. Malashevich and I. Souza, Phys. Rev. B: Condens. Matter Mater. Phys., 2010, 82, 245118.

39 J. E. Sipe and A. I. Shkrebtii, Phys. Rev. B: Condens. Matter Mater. Phys., 2000, 61, 5337-5352.

40 L. Elcoro, B. Bradlyn, Z. Wang, M. G. Vergniory, J. Cano, C. Felser, B. A. Bernevig, D. Orobengoa, G. de la Flor and M. I. Aroyo, J. Appl. Crystallogr., 2017, 50, 1457-1477. 
41 C. J. Bradley, A. P. Cracknell, The Mathematical Theory of Symmetry in Solids: Representation Theory for Point Groups and Space Groups, Oxford University Press, Oxford, New York, 2010.

42 S. Bravo, J. Correa, L. Chico and M. Pacheco, Sci. Rep., 2019, 9, 12754.

43 K. Zhao, X. Li, S. Wang and Q. Wang, Phys. Chem. Chem. Phys., 2019, 21, 246-251.

44 S. Yuan, Q. Zhou, Q. Wu, Y. Zhang, Q. Chen, J.-M. Hou and J. Wang, npj 2D Mater. Appl., 2017, 1, 29.

45 G. Chang, B. J. Wieder, F. Schindler, D. S. Sanchez, I. Belopolski, S.-M. Huang, B. Singh, D. Wu, T.-R. Chang, T. Neupert, S.-Y. Xu, H. Lin and M. Z. Hasan, Nat. Mater., 2018, 17, 978-985.

46 J. F. Nye, Physical Properties of Crystals: Their Representation by Tensors and Matrices, Oxford University Press, Oxford, New York, 1985.

47 M. I. Aroyo, A. Kirov, C. Capillas, J. M. Perez-Mato and H. Wondratschek, Acta Crystallogr., Sect. A: Found. Crystallogr., 2006, 62, 115-128.

48 L. Liu and H. L. Zhuang, Comput. Mater. Sci., 2019, 166, 105-110.

49 M. S. Dresselhaus, G. Dresselhaus and A. Jorio, Group Theory: Application to the Physics of Condensed Matter, Springer-Verlag, Berlin Heidelberg, 2008.

50 D. Vanderbilt, Berry Phases in Electronic Structure Theory: Electric Polarization, Orbital Magnetization and Topological Insulators, Cambridge University Press, 2018.

51 J. Hu, S.-Y. Xu, N. Ni and Z. Mao, Annu. Rev. Mater. Res., 2019, 49, 207-252.

52 N. Nagaosa, T. Morimoto and Y. Tokura, Nat. Rev. Mater., 2020, 5, 621-636.

53 Z. Wang, Y. Sun, X.-Q. Chen, C. Franchini, G. Xu, H. Weng, X. Dai and Z. Fang, Phys. Rev. B: Condens. Matter Mater. Phys., 2012, 85, 195320.

54 Y.-M. Xie, X.-J. Gao, X. Y. Xu, C.-P. Zhang, J.-X. Hu and K. T. Law, 2020, arXiv:2008.03967 [cond-mat].

55 C. Fang, M. J. Gilbert, X. Dai and B. A. Bernevig, Phys. Rev. Lett., 2012, 108, 266802.

56 S. S. Tsirkin, I. Souza and D. Vanderbilt, Phys. Rev. B, 2017, 96, 045102.

57 M. El-Batanouny and F. Wooten, Symmetry and Condensed Matter Physics: A Computational Approach, Cambridge University Press, 2008.

58 D. S. Sanchez, I. Belopolski, T. A. Cochran, X. Xu, J.-X. Yin, G. Chang, W. Xie, K. Manna, V. Süß, C.-Y. Huang, N. Alidoust, D. Multer, S. S. Zhang, N. Shumiya, X. Wang, G.-Q. Wang, T.-R. Chang, C. Felser, S.-Y. Xu, S. Jia, H. Lin and M. Z. Hasan, Nature, 2019, 567, 500-505.

59 F. Flicker, F. de Juan, B. Bradlyn, T. Morimoto, M. G. Vergniory and A. G. Grushin, Phys. Rev. B, 2018, 98, 155145.

60 M.-A. Sánchez-Martínez, F. de Juan and A. G. Grushin, Phys. Rev. B, 2019, 99, 155145.

61 Y.-J. Jin, R. Wang, J.-Z. Zhao, Y.-P. Du, C.-D. Zheng, L.-Y. Gan, J.-F. Liu, H. Xu and S. Y. Tong, Nanoscale, 2017, 9, 13112-13118.
62 J. Ahn, D. Kim, Y. Kim and B.-J. Yang, Phys. Rev. Lett., 2018, 121, 106403.

63 S. Li, Y. Liu, S.-S. Wang, Z.-M. Yu, S. Guan, X.-L. Sheng, Y. Yao and S. A. Yang, Phys. Rev. B, 2018, 97, 045131.

64 C. Zhong, W. Wu, J. He, G. Ding, Y. Liu, D. Li, S. A. Yang and G. Zhang, Nanoscale, 2019, 11, 2468-2475.

65 W. Wu, Y. Jiao, S. Li, X.-L. Sheng, Z.-M. Yu and S. A. Yang, Phys. Rev. Mater., 2019, 3, 054203.

66 M. Sakano, M. Hirayama, T. Takahashi, S. Akebi, M. Nakayama, K. Kuroda, K. Taguchi, T. Yoshikawa, K. Miyamoto, T. Okuda, K. Ono, H. Kumigashira, T. Ideue, Y. Iwasa, N. Mitsuishi, K. Ishizaka, S. Shin, T. Miyake, S. Murakami, T. Sasagawa and T. Kondo, Phys. Rev. Lett., 2020, 124, 136404.

67 B. Bradlyn, J. Cano, Z. Wang, M. G. Vergniory, C. Felser, R. J. Cava and B. A. Bernevig, Science, 2016, 353, aaf5037.

68 C. Shekhar, A. K. Nayak, Y. Sun, M. Schmidt, M. Nicklas, I. Leermakers, U. Zeitler, Y. Skourski, J. Wosnitza, Z. Liu, Y. Chen, W. Schnelle, H. Borrmann, Y. Grin, C. Felser and B. Yan, Nat. Phys., 2015, 11, 645-649.

69 X. Huang, L. Zhao, Y. Long, P. Wang, D. Chen, Z. Yang, H. Liang, M. Xue, H. Weng, Z. Fang, X. Dai and G. Chen, Phys. Rev. X, 2015, 5, 031023.

70 S. Wang, B.-C. Lin, A.-Q. Wang, D.-P. Yu and Z.-M. Liao, Adv. Phys.: X, 2017, 2, 518-544.

71 S. S. Tsirkin, P. A. Puente and I. Souza, Phys. Rev. B, 2018, 97, 035158.

72 Z. K. Liu, L. X. Yang, Y. Sun, T. Zhang, H. Peng, H. F. Yang, C. Chen, Y. Zhang, Y. F. Guo, D. Prabhakaran, M. Schmidt, Z. Hussain, S. K. Mo, C. Felser, B. Yan and Y. L. Chen, Nat. Mater., 2016, 15, 27-31.

73 S. Nishihaya, M. Uchida, Y. Nakazawa, M. Kriener, Y. Kozuka, Y. Taguchi and M. Kawasaki, Sci. Adv., 2018, 4, eaar5668.

74 J. Ibañez-Azpiroz, S. S. Tsirkin and I. Souza, Phys. Rev. B, 2018, 97, 245143.

75 A. Pospischil and T. Mueller, Appl. Sci., 2016, 6, 78.

76 G. Long, R. Sabatini, M. I. Saidaminov, G. Lakhwani, A. Rasmita, X. Liu, E. H. Sargent and W. Gao, Nat. Rev. Mater., 2020, 5, 423-439.

77 E. S. Morell, L. Chico and L. Brey, 2D Mater., 2017, 4, 035015.

78 Z. Addison, J. Park and E. J. Mele, Phys. Rev. B, 2019, 100, 125418.

79 L. Z. Tan, F. Zheng, S. M. Young, F. Wang, S. Liu and A. M. Rappe, npj Comput. Mater., 2016, 2, 16026.

80 Q. Xu, Y. Zhang, K. Koepernik, W. Shi, J. van den Brink, C. Felser and Y. Sun, npj Comput Mater, 2020, 6, 32.

81 C. Le, Y. Zhang, C. Felser and Y. Sun, Phys. Rev. B, 2020, 102, 121111.

82 Z. Ni, B. Xu, M. Á. Sánchez-Martínez, Y. Zhang, K. Manna, C. Bernhard, J. W. F. Venderbos, F. de Juan, C. Felser, A. G. Grushin and L. Wu, npj Quantum Mater., 2020, $5,96$.

83 A. M. Cook, B. M. Fregoso, F. de Juan, S. Coh and J. E. Moore, Nat. Commun., 2017, 8, 14176. 
84 S. V. Gallego, J. Etxebarria, L. Elcoro, E. S. Tasci and J. M. Perez-Mato, Acta Crystallogr., Sect. A: Found. Adv., 2019, 75, 438-447.

85 T. Rangel, B. M. Fregoso, B. S. Mendoza, T. Morimoto, J. E. Moore and J. B. Neaton, Phys. Rev. Lett., 2017, 119, 067402.

86 Y. Zhang, H. Ishizuka, J. van den Brink, C. Felser, B. Yan and N. Nagaosa, Phys. Rev. B, 2018, 97, 241118.

87 Y. Zhang, F. de Juan, A. G. Grushin, C. Felser and Y. Sun, Phys. Rev. B, 2019, 100, 245206.
88 S. M. Young and A. M. Rappe, Phys. Rev. Lett., 2012, 109, 116601.

89 S. M. Young, F. Zheng and A. M. Rappe, Phys. Rev. Lett., 2012, 109, 236601.

90 A. Zenkevich, Y. Matveyev, K. Maksimova, R. Gaynutdinov, A. Tolstikhina and V. Fridkin, Phys. Rev. B: Condens. Matter Mater. Phys., 2014, 90, 161409.

91 G. Bir and G. Pikus, Symmetry and Strain-induced Effects in Semiconductors, Wiley, 1974. 